\title{
p38 mitogen-activated protein kinase inhibition modulates nucleus pulposus cell apoptosis in spontaneous resorption of herniated intervertebral discs: An experimental study in rats
}

\author{
YU ZHU ${ }^{1,2^{*}}$, JIN-TAO LIU ${ }^{2 *}$, LI-YAN YANG ${ }^{3}$, WEN-PEI DU ${ }^{3}$, XIAO-CHUN LI ${ }^{2}$, \\ XIANG QIAN $^{2}$, PENG-FEI YU ${ }^{2}$, JIAN-WEN LIU ${ }^{3}$ and HONG JIANG ${ }^{2}$ \\ ${ }^{1}$ Graduate School of Shanghai University of Traditional Chinese Medicine, Shanghai 201203; \\ ${ }^{2}$ Department of Orthopaedic Surgery, Suzhou Hospital of Traditional Chinese Medicine, Suzhou, \\ Jiangsu 215009; ${ }^{3}$ State Key Laboratory of Bioreactor Engineering and School of Pharmacy, \\ East China University of Science and Technology, Shanghai 200237, P.R. China
}

Received May 27, 2015; Accepted March 9, 2016

DOI: $10.3892 / \mathrm{mmr} .2016 .5039$

\begin{abstract}
The present study was performed to investigate the role of p38 mitogen-activated protein kinase (MAPK) in the resorption of herniated intervertebral discs in 30 rats. In the non-contained and p38 MAPK inhibition (p38i) groups, two coccygeal intervertebral discs (IVDs) were removed and wounded prior to relocation into the subcutaneous space of the skin of the back. In the contained group, the cartilage endplates maintained their integrity. Furthermore, SB203580 was injected intraperitoneally into the p38i group, whereas saline was injected into the other two groups. In the non-contained group, the weight of the relocated IVDs decreased to a greater extent over time when compared with the contained and p38i groups. Phosphorylated p38, tumor necrosis factor- $\alpha$, and interleukin-1 $\beta$ were observed to exhibit higher expression levels in the non-contained group compared with the contained and $\mathrm{p} 38 \mathrm{i}$ groups, at weeks 1 and 4 post-surgery. The expression level of caspase-3 and the densities of apoptotic disc cells were significantly higher in the non-contained group compared with the contained and p38i groups at 4 weeks post-surgery. In conclusion, p38 MAPK induces apoptosis in IVDs, while also
\end{abstract}

Correspondence to: Dr Hong Jiang, Department of Orthopaedic Surgery, Suzhou Hospital of Traditional Chinese Medicine, 889 Wuzhongxi Road, Suzhou, Jiangsu 215009, P.R. China

E-mail: doctorhong@yeah.net

Dr Jian-Wen Liu, State Key Laboratory of Bioreactor Engineering and School of Pharmacy, East China University of Science and Technology, 130 Meilong Road, Shanghai 200237, P.R. China E-mail: liujanwen@163.com

*Contributed equally

Key words: intervertebral disc herniation, spontaneous resorption, p38 mitogen-activated protein kinase, apoptosis accelerating the resorption of the relocated IVDs. Thus, p38 MAPK may be important in spontaneous resorption of IVDs.

\section{Introduction}

Intervertebral disc (IVD) herniation is the major cause of chronic sciatica and lower back pain (1). However, these severe symptoms can be noticeably relieved in $70 \%$ of lumbar disc herniation patients within 6 weeks of onset, while certain patients demonstrate a decrease in the size, or disappearance, of the herniated disc by magnetic resonance imaging (MRI) and computed tomography (CT) (1-3). This natural resorption is more likely to occur in extruded types, particularly in the sequestered type, as the herniated disc is more easily exposed to the epidural vascular supply by the presence of a tear in the posterior longitudinal ligament (PLL) $(1,4,5)$. The mechanism of spontaneous resorption is associated with numerous factors. It has been shown that infiltrating macrophages and newly formed vessels promote the progression of spontaneous herniated disc resorption (6). Tumor necrosis factor- $\alpha$ (TNF- $\alpha$ ) and interleukin-1 $\beta$ (IL-1 $\beta$ ), which are released from macrophages after the onset of disc herniation, are crucial in herniated disc resorption $(7,8)$.

The apoptosis of IVD cells is increased more in non-contained disc herniation than in contained disc herniation, in which the herniated nucleus pulposus penetrates the PLL and is exposed to the epidural space (9). p38 mitogen-activated protein kinase (MAPK) is hypothesized to be closely associated with inflammation and apoptosis $(10,11)$. Furthermore, increasing evidence suggests that activated p38 MAPK induces apoptosis in the herniated disc $(12,13)$. However, the association between p38 MAPK and apoptosis in herniated disc resorption remains to be clarified, thus the present study hypothesized that the induction of herniated disc apoptosis by p38 MAPK activation may be significant in spontaneous resorption.

In the current study, the occurrence of apoptosis in disc cells and the expression level of caspase-3 was examined in a rat model of IVD herniation. In addition, the expressions of 
TNF- $\alpha$, IL-1 $\beta$, p38 MAPK, and P-p38 (phosphorylated p38) was investigated. These data may provide further insight into the underlying mechanism of spontaneous resorption of lumbar disc herniation.

\section{Materials and methods}

Animals and materials. All experiments were approved by the Institutional Animal Care and Use Committee (School of Pharmacy, East China University of Science and Technology; Shanghai, China). A total of 30 male Sprague-Dawley rats, (weight, 230-300 g) were obtained from the Shanghai Laboratory Animal Center Laboratory Animal Co., Ltd. (Shanghai, China). SB203580 (a p38 MAPK inhibitor) was obtained from Selleck Chemicals Co., Ltd. (Houston, TX, USA). The following antibodies were used: Rabbit anti-human polyclonal IL-1 $\beta$ (1:1,000; 13082-1-AP; Proteintech Group, Inc., Chicago, IL, USA) and polyclonal rabbit anti-human P-p38 (1:1,000; GWB-ASB336; GenWay Biotech, Inc., San Diego, CA, USA), rabbit anti-human polyclonal p38 MAPK (1:1,000; 33149; Signalway Antibody Co., Ltd., Maryland, MD, USA), rabbit anti-human polyclonal TNF- $\alpha$ (1:1,000; 17590-1-AP; Proteintech Group, Inc.) and rabbit anti-human polyclonal caspase-3 (1:1,000; 19677-1-AP; Proteintech Group, Inc.).

Animal model. The 30 rats were divided into control and p38i (p38 MAPK inhibition) groups in a 2:1 ratio. The rats were housed separately in plastic cages in a pathogen-free environment. The rats were fed sterile feed (Shanghai SLAC Laboratory Animal Co., Ltd., Shanghai, China) and were maintained under a $12 \mathrm{~h}$ light/dark cycle. The control group was subdivided equally into contained and non-contained groups according to different processing of the discs. Two coccygeal IVDs, containing the nucleus pulposus, annulus fibrosus and adjacent cartilage endplates, were obtained under $40 \mathrm{mg} / \mathrm{kg}$ intraperitoneal pentobarbital (Sigma-Aldrich, St. Louis, MO, USA) from the rat tail. In the non-contained group, the cartilage endplates were punctured with a needle and the harvested disc material was weighed using the FA1004B Millionth Sophisticated Analytical Balance (Shanghai Precision Instrument Co., Ltd., Shanghai, China) prior to autografting into the back muscle of the rat. In the contained group, the discs were placed into the back directly after noting the weight. In the p38i group, rats with autografted non-contained discs were injected into the peritoneum, with $10 \mathrm{mg} / \mathrm{kg} \mathrm{SB} 203580$, daily from days 1 to 28 after surgery. In the other two groups, rats received an injection of the same quantity of saline. Five rats from each group were sacrificed by overdose with pentobarbital sodium $(200 \mathrm{mg} / \mathrm{kg})$ for harvested disc material at weeks 1 and 4 post-surgery.

Tissue processing. After recording the weight of the harvested discs, one tissue specimen from each rat was prepared for histological observation, immunohistochemistry and terminal deoxynucleotidyl transferase dUTP nick end labeling (TUNEL) staining. All samples were fixed in $10 \%$ neutral buffered formalin (YiYan Biological Technology, Ltd., Shanghai, China) at room temperature overnight and embedded in paraffin (YiYan Biological Technology, Ltd.). The tissue specimens were sliced into $5 \mathrm{~mm}$-thick paraffin sections in the axial plane, using a microtome (BZ-600; BZ Technology Co., Ltd., Daventry, UK).
Hematoxylin and eosin (H\&E; Qianchen Biological Technology Co., Ltd., Shanghai, China) staining was used according to the standard method and the morphology of the harvested discs was examined under a microscope (170BN; Wincom Company Ltd., Changsha, China). The remaining tissue specimens were prepared for western blot by mechanically pulverizing the tissue with a pestle and mortar on ice and homogenizing in phosphate-buffered saline (PBS; Sigma-Aldrich).

Western blot analysis. Protein concentrations were determined using the bicinchoninic acid assay (Thermo Fisher Scientific, Inc., Waltham, MA, USA). Proteins $(50 \mu \mathrm{g})$ were resolved in $10 \%$ sodium dodecyl sulfate-polyacrylamide gels (Beyotime Institute of Biotechnology, Shanghai, China), then transferred onto nitrocellulose membranes (GE Healthcare Life Sciences, Uppsala, Sweden). The membrane was blocked with 5\% nonfat milk in Tris-buffered saline (Cell Signaling Technology, Inc., Danvers, MA, USA) for $1 \mathrm{~h}$ at room temperature and incubated with the IL-1 $\beta$, TNF- $\alpha$, p38 and P-p38 primary antibodies in dilution buffer (Beyotime Institute of Biotechnology) overnight at $4^{\circ} \mathrm{C}$. The membranes were incubated with the goat anti-rabbit polyclonal secondary antibody (1:10,000; 110806; Jackson ImmunoResearch Laboratories, Inc., West Grove, PA, USA), alkaline phosphatase (AP; Roche Diagnostics, Basel, Switzerland), conjugated with AP containing nitro-blue tetrazolium chloride/5-bromo-4-chloro-3-indolyl-phosphate [Meryer (Shanghai) Chemical Technology Co., Ltd., Shanghai, China] at room temperature for 10-20 min, and imaged using enhanced chemiluminescence (GE Healthcare Life Sciences, Piscataway, NJ, USA). The X-ray films (Kodak, Rochester, NY, USA) were scanned, and then the intensity of each signal density was measured and analyzed using ImageJ software, version 1.48 (National Institutes of Health, Bethesda, MD, USA). $\beta$-actin served as the internal control for protein loading.

Immunohistochemical staining. The tissue specimens were dewaxed with xylene (Qianchen Biological Technology Co., Ltd.) and rehydrated using a graded alcohol series. The endogenous peroxidase reactions were quenched with $3 \%$ $\mathrm{H}_{2} \mathrm{O}_{2}$ (Qianchen Biological Technology Co., Ltd.) for $10 \mathrm{~min}$ at room temperature. Then, nonspecific binding was blocked with 5\% normal bovine serum albumin [Meryer (Shanghai) Chemical Technology Co., Ltd.] for $1 \mathrm{~h}$ at room temperature. The specimens were washed three times with PBS after incubation with the anti-caspase-3 primary antibodies overnight at $4^{\circ} \mathrm{C}$. After incubation for $2 \mathrm{~h}$ with fluorescent-labeled secondary antibodies, the specimens were washed another three times with PBS. Subsequently, for marker staining, the specimens were incubated with streptavidin-horseradish peroxidase [Meryer (Shanghai) Chemical Technology Co., Ltd.] at room temperature for $2 \mathrm{~h}$ and subsequently immersed in 3,3'-diaminobenzidine tetrachloride (Roche Diagnostics) in the dark for 5-10 min. After counterstaining with hematoxylin, the specimens were dehydrated with a graded series of alcohol and examined under a light microscope (LB202; Leader Precision Instrument Co., Ltd., Dongguan, China).

TUNEL staining. The tissue specimens were rehydrated and endogenous peroxidase reactions were quenched (as mentioned above) and incubated with proteinase K (Roche Diagnostics) 

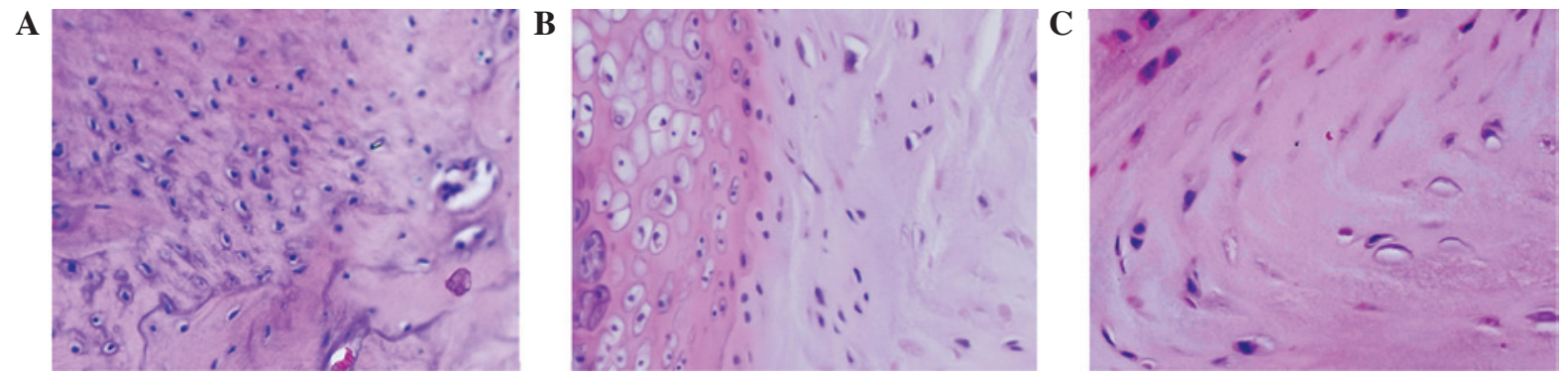

Figure 1. Hematoxylin and eosin-stained sections were examined under a microscope four weeks after surgery (magnification, x200). (A) Numerous newly formed vessels and macrophages were observed in the relocated discs in the non-contained group and, in particular, the morphological structure was clearly destroyed, exhibiting disordered annulus fibrosus and shrinking of the nucleus pulposus tissue. (B) In the p38 inhibition group, the disc structure exhibited mild morphological alterations, and newly formed vessels and macrophages were observed in the relocated discs. (C) There was almost no change in the structure of the relocated discs in the contained group, furthermore, newly formed vessels and infiltrated macrophages were not observed.

for $15 \mathrm{~min}$ at $37^{\circ} \mathrm{C}$. The tissue specimens were then incubated with Equilibration Buffer (Roche Diagnostics) for $10 \mathrm{~min}$ prior to incubation with BrightGreen Labeling Mix (Roche Diagnostics) and TUNEL (Roche Diagnostics), for $1 \mathrm{~h}$ in the dark. Subsequent to three washes with distilled water, the tissue specimens were examined for apoptosis under a fluorescence microscope (LF302; Leader Precision Instrument Co., Ltd.).

Statistical analysis. Differences in the weight of the relocated discs were determined by one-way analysis of variance, followed by the Bonferroni post hoc test for multiple comparisons. Differences between any two groups were analyzed using the unpaired Student's $t$-test or the Mann-Whitney test as appropriate and $\mathrm{P}<0.05$ was considered to indicate a statistically significant difference. SPSS software, version 18.0 (SPSS, Inc., Chicago, IL, USA) was used for statistical analysis.

\section{Results}

Histological changes. H\&E-stained sections were examined under a microscope. As shown in Fig. 1, morphological changes in the annulus fibrosus and eosinophilic staining were not observed in contained disc tissues, but were apparent in the p38i group and were more evident in the non-contained group. The extracellular matrix and collagen fibers were disordered in the non-contained group, and were accompanied by neovascularization and inflammatory cell infiltration, including the presence of macrophages in the relocated discs.

Weight change. The weight of relocated discs was examined prior to disc cell relocation and following sacrifice. The weight in the non-contained group decreased significantly at week 4 compared with week 1 and the time of surgery $(\mathrm{P}<0.05)$. However, in the contained and p38i groups (Fig. 2), there was no significant difference in disc weight between any of the three time-points. This indicated that the weight of the relocated disc in the non-contained group decreased more markedly than the other two groups as time progressed.

TNF- $\alpha$ and IL-1 $\beta$ expression levels. The expression levels of TNF- $\alpha$ and IL-1 $\beta$ were observed in the three groups by western blot analysis (Fig. 3). The expression levels of TNF- $\alpha$ and IL-1 $\beta$ were markedly higher in the non-contained and p38i groups compared with the contained group at week 1 , however,

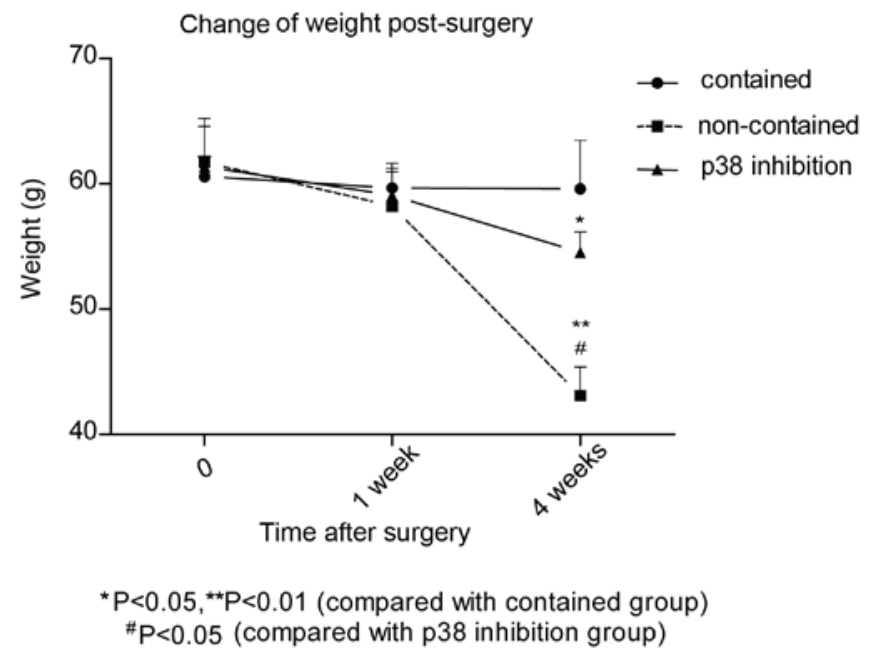

Figure 2. Change in weight of relocated discs. At the time of surgery, and at weeks 1 and 4 post-surgery, the weight of each relocated disc was measured. In the non-contained group, the weight gradually decreased, and a significant difference was observed when compared with the contained and p38 inhibition groups at week 4 . This difference was also observed between the p38 inhibition and contained groups. ${ }^{*} \mathrm{P}<0.05,{ }^{* *} \mathrm{P}<0.01$ vs. contained; ${ }^{\text {"}} \mathrm{P}<0.05$ vs. $\mathrm{p} 38$ inhibition.

at week 4 the difference was reduced. In the p38i group, the expression levels of TNF- $\alpha$ and IL- $1 \beta$ decreased gradually over time.

Activation of $p 38$ MAPK. A high expression level of P-p38 was observed in the non-contained group, indicating that p38 phosphorylation was significantly suppressed by p38i. However, there was almost no difference in the expression intensity of non-P-p38 between all three groups at weeks 1 and 4 (Fig. 3).

Apoptosis of nucleus pulposus cells. TUNEL staining was performed to detect the presence of apoptotic cells in the relocated discs. As shown in Fig. 4, the apoptotic percentage differed between each group and was highest in the non-contained group. Apoptosis in the p38i group was less than that in the non-contained group, however, was more than in the contained group.

Caspase-3 expression. Immunohistochemical staining was performed to evaluate the expression of caspase- 3 


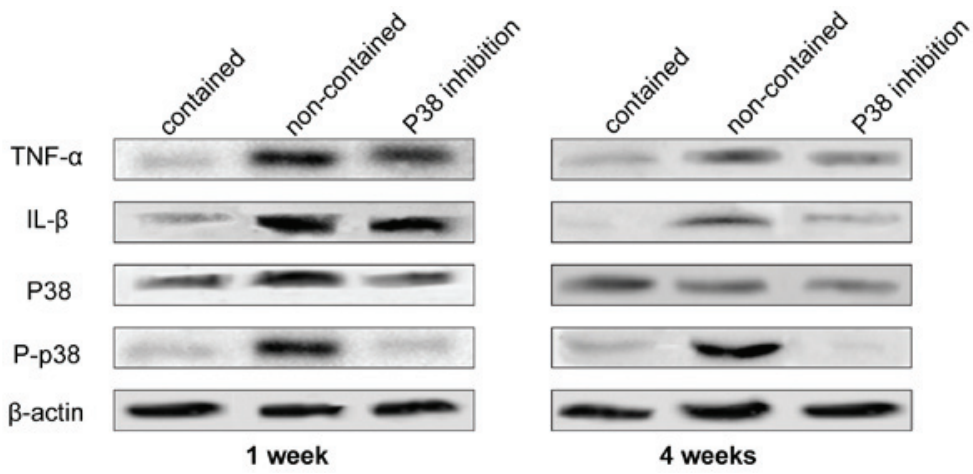

Figure 3. Western blots were generated and probed for TNF- $\alpha$, IL-1 1 , p38 MAPK, and p-p38 MAPK at weeks 1 and 4 . In the non-contained group, TNF- $\alpha$, IL-1 $\beta$, and p38 MAPK exhibited high expression levels, which were not observed in the contained group. The expression level of p38 MAPK was inhibited in the presence of SB203580, indicating that TNF- $\alpha$ and IL-1 $\beta$ promoted $\mathrm{p} 38$ expression in this condition. Additionally, the expression levels of TNF- $\alpha$ and IL-1 $\beta$ were suppressed by SB203580 treatment. TNF- $\alpha$, tumor necrosis factor- $\alpha$; IL-1 $\beta$, interleukin-1 $\beta$; MAPK, mitogen-activated protein kinase; P, phosphorylated.
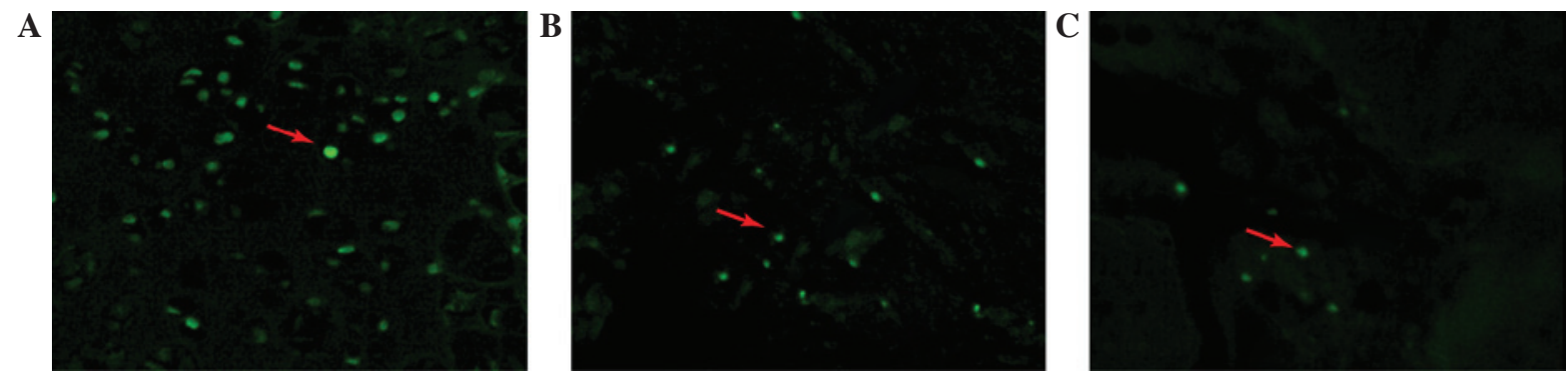

Figure 4. Relocated discs in the (A) non-contained (B) p38 inhibition and (C) contained groups were stained for apoptosis using the terminal deoxynucleotidyl transferase dUTP nick end labeling method; green fluorescence indicates apoptotic disc cells. The apoptotic percentage was highest in the non-contained group, followed by the p38 inhibition group, and the lowest was observed in the contained group. Arrows indicate apoptotic cells (magnification, x200).
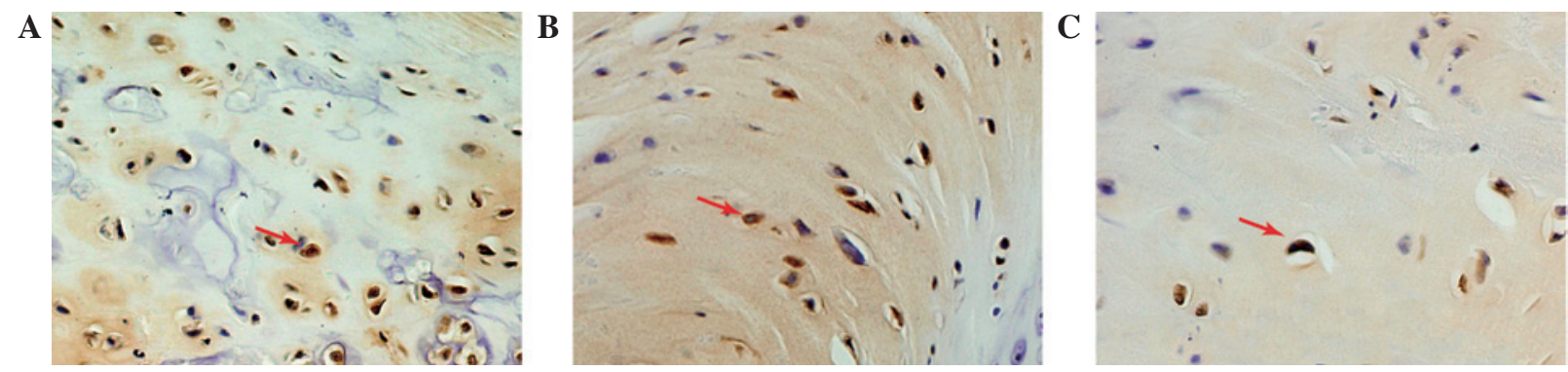

Figure 5. Typical appearance of immunoreactive cells for proteinases (magnification, x200). Immunohistochemical staining for caspase-3 in the (A) non-contained, (B) p38 inhibition and (C) contained groups. Caspase-3 immunoreactivity is indicated by brown-yellow staining (indicated by red arrows).

in the relocated discs. Caspase-3 staining demonstrated different trends in the three groups (Fig. 5). The majority of the caspase-3-positive disc cells were observed in the non-contained group disc tissue. The expression of caspase-3 in the p38i group was higher than in the contained group.

\section{Discussion}

With developments in imaging techniques, such as MRI and $\mathrm{CT}$, there has been an increase in the reporting of the disappearance or decreases in size of herniated IVDs (14). Regression is more significant in extruded and sequestrated herniated discs. Disc migration is a subtype of disc extrusion, where the herniation is exposed to the epidural space, as well as transligamentous herniation. Komori et al (1) identified that the complete resolution rate was higher in a migration group when compared with a non-migration group (41 vs. 0\%). Various hypotheses have been proposed to explain the mechanism by which spontaneous resorption occurs. One hypothesis is that, in disc bulges and protrusions, the herniation may retract back into the parent disc $(15,16)$. A second is that dehydration promotes disc regression due to a higher MRI T2 signal intensity, as higher regression rates have previously been reported $(17,18)$. Disc herniation into the epidural space causes an inflammatory reaction and neovascularization, resulting in the absorption of the herniated disc by phagocytosis and enzymatic degradation (19). In addition to these hypotheses, the apoptosis of disc cells has received greater attention, as evidence indicates that a higher degree of apoptotic disc cells is present in non-contained discs when compared with 
contained discs, suggesting that apoptosis of disc cells may be another mechanism in spontaneous resorption (20).

In the current study, an experimental rat model of disc resorption was proposed, which was modified based on a previously described method $(21,22)$. The changes in disc weight and morphological structure indicated that this model appropriately simulated the sequestrated type of human disc herniation, in which spontaneous resorption is most likely to occur. In the non-contained model, newly formed vessels and macrophages easily infiltrated into the exposed disc tissues when the cartilage endplate was punctured with a needle. However, the infiltration was not obvious in the contained model, as the disc tissue is isolated from the blood supply and immune system. The decrease in weight of the relocated IVD may indicate spontaneous regression in vivo $(8,23)$, thus this decrease demonstrated that the needle puncture model adopted was effective, simple and practical in the mechanistic investigation of spontaneous regression by simulating extruded and sequestrated intervertebral disc herniation.

The MAPK signaling pathway family acts as a major kinase pathway, which regulates numerous physiological activities in cells, such as inflammation, metabolic balance, and apoptosis (24). Previous studies have demonstrated that when the IVD was relocated in vivo, the autografts induced TNF- $\alpha$ and IL-1 $\beta$ mRNA upregulation, rapidly followed by macrophage infiltration (25). The phosphorylation of p38 MAPK, an important component of the MAPK family, was closely associated with the secretion and accumulation of proinflammatory factors. p38 MAPK can be activated by TNF- $\alpha$ and IL- $1 \beta$ through numerous signaling pathways, such as apoptosis-stimulating kinase and transforming growth factor- $\beta$-activated kinase (26-28). P-p38 MAPK in nucleus pulposus cells was markedly increased after cells were exposed to TNF- $\alpha$ or IL-1 $\beta$, and the mRNA expression levels of TNF- $\alpha$ and IL-1 $\beta$ were downregulated by p38 inhibition $(29,30)$. Furthermore, IL-1 $\beta$ and TNF- $\alpha$ may stimulate herniated disc nucleus pulposus cells to produce prostaglandin E2, IL-6 and matrix metalloproteinase-3 (MMP-3), which were closely associated with disc degeneration, but were decreased when p38 MAPK was inhibited. In a previous study, p38 MAPK inhibition increased the ratio of tissue inhibitor of metalloproteinases metallopeptidase inhibitor 1 to MMP-3 in vitro when activated by IL-1 $\beta$ or TNF- $\alpha$, subsequently influencing the degradation of the extracellular matrix of nucleus pulposus cells (29).

In the present study, the expression levels of TNF- $\alpha$ and IL-1 $\beta$ were observed after the IVD was implanted in the non-contained group, accompanied by the high expression level of P-p38 MAPK. However, in the contained group, these proinflammatory factors were almost undetectable, which may have been due to the resulting isolation between the relocated disc, and the now segregated blood supply. Furthermore, P-p38 MAPK was expressed at a moderately low level. In addition, the current study demonstrated that the P-p38 MAPK was suppressed significantly by SB203580 in the p38i group. It has been previously shown that p38 MAPK is activated by TNF- $\alpha$ and IL-1 $\beta$ secretion from macrophages or IVD tissue when the herniated disc penetrates the PLL (31).

p38 MAPK induces apoptosis through various signaling pathways. Cai et al (12) found that the phosphorylation of
Bim $_{\mathrm{EL}}$, a member of the Bcl-2 family, on Ser-65 may be a common regulatory point for cell death induced by the c-Jun N-terminal kinase and p38 MAPK signaling pathways. Hsu et al (32) proposed that receptor engagement activates p38 $\alpha$ to promote apoptosis by the induction of Fas ligand (FasL) expression. In addition, p38 MAPK activation induces the activation of caspases, such as caspase-3, and apoptosis via the Fas-mediated death pathway (33). Regarding disc cells, Rannou et al (13) suggested that p38 MAPK signaling is crucial in the process of annulus fibrosus cell apoptosis during mechanical overload. Apoptotic cells are induced by the Fas-mediated death pathway, in which p38 $\alpha$ MAPK activation increases the expression of Fas and FasL proteins, as well as caspase activation (33). In herniated discs, the apoptosis of disc cells following herniation differs depending on the type of herniation, which is higher in non-contained discs when compared with contained discs (9). When IVD cells undergo apoptosis, they are phagocytosed by macrophages and disc cells, including neighboring cells within cell clusters (34).

The present study found that the expression ratio of apoptotic cells and caspase- 3 in the non-contained group was significantly higher than that of the contained group, however, this advantage was suppressed by p38 MAPK inhibition. Thus, following activation by TNF- $\alpha$ and IL-1 $\beta$, P-p38 MAPK induces apoptosis of disc cells via a mechanism that remains unknown.

In conclusion, p38 MAPK was found to be involved in the process of spontaneous resorption by the induction of apoptosis in disc cells in a rat model of IVD herniation. To the best of our knowledge, this finding is the first direct evidence of the involvement of $\mathrm{p} 38 \mathrm{MAPK}$ in spontaneous resorption of IVDs.

\section{Acknowledgements}

The present study was supported by the National Natural Science Funds of China (grant no. 81473691).

\section{References}

1. Komori H, Shinomiya K, Nakai O, Yamaura I, Takeda S and Furuya K: The natural history of herniated nucleus pulposus with radiculopathy. Spine (Phila Pa 1976) 21: 225-229, 1996.

2. Yu PF, Jiang FD, Liu JT and Jiang H: Outcomes of conservative treatment for ruptured lumbar disc herniation. Acta Orthop Belg 79: 726-730, 2013.

3. Haro H: Translational research of herniated discs: Current status of diagnosis and treatment. J Orthop Sci 19: 515-520, 2014.

4. Maigne JY, Rime B and Deligne B: Computed tomographic follow-up study of forty-eight cases of nonoperatively treated lumbar intervertebral disc herniation. Spine (Phila Pa 1976) 17: 1071-1074, 1992

5. Ahn SH, Ahn MW and Byun WM: Effect of the transligamentous extension of lumbar disc herniations on their regression and the clinical outcome of sciatica. Spine (Phila Pa 1976) 25: 475-480, 2000.

6. Minamide A, Tamaki T, Hashizume H, Yoshida M, Kawakami M and Hayashi N: Effects of steroid and lipopolysaccharide on spontaneous resorption of herniated intervertebral discs: An experimental study in the rabbit. Spine (Phila Pa 1976) 23: 870-876, 1998.

7. Haro H, Crawford HC, Fingleton B, Shinomiya K, Spengler DM and Matrisian LM: Matrix metalloproteinase-7-dependent release of tumor necrosis factor-alpha in a model of herniated disc resorption. J Clin Invest 105: 143-150, 2000. 
8. Yoshida M, Nakamura T, Sei A, Kikuchi T, Takagi K and Matsukawa A: Intervertebral disc cells produce tumor necrosis factor alpha, interleukin-lbeta and monocyte chemoattractant protein-1 immediately after herniation: An experimental study using a new hernia model. Spine (Phila Pa 1976) 30: 55-61, 2005 .

9. Ha KY, Koh IJ, Kirpalani PA, Kim YY, Cho YK, Khang GS and Han CW: The expression of hypoxia inducible factor-1alpha and apoptosis in herniated discs. Spine (Phila Pa 1976) 31: 1309-1313, 2006.

10. Lee JC, Laydon JT, McDonnell PC, Gallagher TF, Kumar S, Green D, McNulty D, Blumenthal MJ, Heys JR and Landvatter SW: A protein kinase involved in the regulation of inflammatory cytokine biosynthesis. Nature 372: 739-746, 1994.

11. Xia Z, Dickens M, Raingeaud J, Davis RJ and Greenberg ME: Opposing effects of ERK and JNK-p38 MAP kinases on apoptosis. Science 270: 1326-1331, 1995.

12. Cai B, Chang SH, Becker EB, Bonni A and Xia Z: p38 MAP kinase mediates apoptosis through phosphorylation of BimEL at Ser-65. J Biol Chem 281: 25215-25222, 2006.

13. Rannou F, Lee TS, Zhou RH, Chin J, Lotz JC, Mayoux-Benhamou MA, Barbet JP, Chevrot A and Shyy JY: Intervertebral disc degeneration: The role of the mitochondrial pathway in annulus fibrosus cell apoptosis induced by overload. Am J Pathol 164: 915-924, 2004.

14. Cvetanovich GL, Hsu AR, Frank RM, An HS and Andersson GB: Spontaneous resorption of a large cervical herniated nucleus pulposus. Am J Orthop (Belle Mead NJ) 43: E140-E145, 2014.

15. Teplick JG and Haskin ME: Spontaneous regression of herniated nucleus pulposus. Am J Roentgenol 145: 371-375, 1985.

16. Sari H, Akarirmak U, Karacan I and Akman H: Computed tomographic evaluation of lumbar spinal structures during traction. Physiother Theory Pract 21: 3-11, 2005.

17. Splendiani A, Puglielli E, De Amicis R, Barile A, Masciocchi C and Gallucci M: Spontaneous resolution of lumbar disk herniation: Predictive signs for prognostic evaluation. Neuroradiology 46 916-922, 2004

18. Henmi T, Sairyo K, Nakano S, Kanematsu Y, Kajikawa T, Katoh S and Goel VK: Natural history of extruded lumbar intervertebral disc herniation. J Med Invest 49: 40-43, 2002

19. Rätsep T, Minajeva A and Asser T: Relationship between neovascularization and degenerative changes in herniated lumbar intervertebral discs. Eur Spine J 22: 2474-2480, 2013.

20. Park JB, Kim KW, Han CW and Chang H: Expression of fas receptor on disc cells in herniated lumbar disc tissue. Spine (Phila Pa 1976) 26: 142-146, 2001.

21. Meng W, Yonenobu K, Ariga K, Nakase T, Okuda S, Obata K and Yoshikawa $\mathrm{H}$ : Localization of cathepsins $\mathrm{G}$ and $\mathrm{L}$ in spontaneous resorption of intervertebral discs in a rat experimental model. J Musculoskelet Neuronal Interact 2: 171-176, 2001.
22. Geiss A, Larsson K, Rydevik B, Takahashi I and Olmarker K: Autoimmune properties of nucleus pulposus: An experimental study in pigs. Spine (Phila Pa 1976) 32: 168-173, 2007.

23. Zhou G, Dai L, Jiang X, Ma Z, Ping J, Li J and Li X: Effects of human midkine on spontaneous resorption of herniated intervertebral discs. Int Orthop 34: 103-108, 2010.

24. Muthuswamy R, Jenkins F, Bovbjerg D and Kalinski P Synergistic induction of cancer-related immunosuppression by $\beta 2$-adrenergic stress mediators and P38MAPK inflammatory pathway. J Immunother Cancer 1 (Suppl 1):191, 2013.

25. Takada T, Nishida K, Maeno K, Kakutani K, Yurube T, Doita M and Kurosaka M: Intervertebral disc and macrophage interaction induces mechanical hyperalgesia and cytokine production in a herniated disc model in rats. Arthritis Rheum 64: 2601-2610, 2012.

26. Kimura N, Matsuo R, Shibuya H, Nakashima $\mathrm{K}$ and Taga T: BMP2-induced apoptosis is mediated by activation of the TAK1-p38 kinase pathway that is negatively regulated by Smad6. J Biol Chem 275: 17647-17652, 2000.

27. Ichijo H, Nishida E, Irie K, ten Dijke P, Saitoh M, Moriguchi T, Takagi M, Matsumoto K, Miyazono K and Gotoh Y: Induction of apoptosis by ASK1, a mammalian MAPKKK that activates SAPK/JNK and p38 signaling pathways. Science 275: 90-94, 1997.

28. Suzuki K, Hino M, Kutsuna H, Hato F, Sakamoto C, Takahashi T, Tatsumi $\mathrm{N}$ and Kitagawa S: Selective activation of p38 mitogen-activated protein kinase cascade in human neutrophils stimulated by IL-1beta. J Immunol 167: 5940-5947, 2001.

29. Studer RK, Aboka AM, Gilbertson LG, Georgescu H, Sowa G, Vo N and Kang JD: P38 MAPK inhibition in nucleus pulposus cells: A potential target for treating intervertebral disc degeneration. Spine (Phila Pa 1976) 32: 2827-2833, 2007.

30. Kakutani K, Pichika R, Yoshikawa T, et al: P38 MAPK inhibition has a positive effect on human and rabbit intervertebral disc degeneration: SP49 (C)/Spine Journal Meeting Abstracts. LWW 131, 2010.

31. Cheng X, Ni B, Zhang Z, Liu Q, Wang L, Ding Y and Hu Y: Polyol pathway mediates enhanced degradation of extracellular matrix via 38 MAPK activation in intervertebral disc of diabetic rats. Connect Tissue Res 54: 118-122, 2012

32. Hsu SC, Gavrilin MA, Tsai MH, Han J and Lai MZ: P38 mitogen-activated protein kinase is involved in Fas ligand expression. J Biol Chem 274: 25769-25776, 1999.

33. Liu WH, Cheng YC and Chang LS: ROS-mediated p38alpha MAPK activation and ERK inactivation responsible for upregulation of Fas and FasL and autocrine Fas-mediated cell death in Taiwan cobra phospholipase A (2)-treated U937 cells. J Cell Physiol 219: 642-651, 2009.

34. Jones P, Gardner L, Menage J, Williams GT and Roberts S: Intervertebral disc cells as competent phagocytes in vitro: Implications for cell death in disc degeneration. Arthritis Res Ther 10: R86, 2008. 